\title{
Preoperative psychological distress no reason to delay total knee arthroplasty: a register-based prospective cohort study of 458 patients
}

\author{
Aamir Mahdi ${ }^{1,2,4}\left(\right.$ D $\cdot$ Maria Hälleberg-Nyman ${ }^{3} \cdot$ Per Wretenberg ${ }^{1,2}$
}

Received: 22 March 2020 / Accepted: 15 July 2020 / Published online: 28 July 2020

(c) The Author(s) 2020

\begin{abstract}
Introduction Total knee arthroplasty (TKA) is effective in alleviating pain and improving function in patients with knee arthritis. Psychological factors are known to affect patient satisfaction after TKA. It is important to determine the effectiveness of TKA in patients with presurgical anxiety and/or depression to avoid excluding them from surgery.

Materials and methods A prospective cohort study was conducted on all patients who underwent TKA during 2016-2018. Patients were divided into four groups: with anxiety, without anxiety, with depression, and without depression. Outcome measures comprised both generic and knee-specific instruments. Each patient group was compared regarding changes in outcome measures one year after surgery. Between-group comparison was also performed.

Results Of the 458 patients with complete data, $15.3 \%$ and $9.6 \%$ had experienced presurgical anxiety and depression, respectively. All patient groups displayed statistical $(P<0.001)$ and clinical improvement in all outcome measures. Patients with presurgical anxiety and/or depression generally displayed less improvement, though the only significant mean differences concerned the Knee Injury and Osteoarthritis Outcome Score (KOOS)-sport score in the non-anxiety and non-depression groups $(P=0.006$ and 0.03 , respectively), a higher proportion of clinically improved KOOS pain in the non-anxiety group $(P=0.03)$, and the general health state in the anxiety and depression groups $(P=0.004$ and 0.04 , respectively).

Conclusions All patients improved in outcome measures 1 year after TKA, regardless of presurgical psychological state. Patients with presurgical anxiety and/or depression benefit greatly from surgery and should not be discriminated against based on presurgical psychological distress, though this fact should not eliminate the preoperative psychological assessment of patients.
\end{abstract}

Level of evidence II.

Keywords Knee arthritis $\cdot$ Knee Injury and Osteoarthritis Outcome Score $\cdot$ Psychological distress $\cdot$ Total knee arthroplasty

Aamir Mahdi

aamir.mahdi@oru.se

Maria Hälleberg-Nyman

maria.halleberg-nyman@oru.se

Per Wretenberg

per.wretenberg@regionorebrolan.se

1 Department of Orthopaedics, Örebro County Council, Örebro, Sweden

2 School of Medical Sciences, Örebro University, Örebro, Sweden

3 School of Health Sciences, Örebro University, Örebro, Sweden

4 Department of Orthopaedics, Faculty of Health and Medicine, Örebro University, SE-701 82 Örebro, Sweden

\section{Introduction}

Total knee arthroplasty (TKA) has proven to be successful in improving pain and function among patients with knee arthritis [1-5]. Unfortunately, there are still patients who are not satisfied after TKA [6-10]. The number of such dissatisfied patients is expected to increase because of an increasing number of patients undergoing TKA [1, 11, 12]. Dissatisfaction rates reported in the literature range between $6 \%$ and $32 \%$, and have been attributed to mechanical, psychological, and combined factors [10, 13-18].

Anxiety is defined as a disorder of persistent and exaggerated feelings of fear in relation to normal situations [19, $20]$. The prevalence of anxiety in the general population as reported in the literature varies, and can be as high as 33\% 
$[19,21]$. Depression is a mental disorder that causes feelings of unhappiness and loss of pleasure in activities once enjoyed [22]. Only $4-8 \%$ of people have a clinical diagnosis of depression; in contrast, symptoms of depression are much more common, but only about one-third of patients with depression symptoms require treatment [23]. A study showed that approximately $20 \%$ of patients with osteoarthritis experience anxiety and/or depression symptoms [24].

Although patients' expectations are the main factor contributing to patient satisfaction after TKA [6, 8, 16, 25-28], the role of psychological factors has been paid more attention in recent years [13, 14, 24, 26, 29-35]. One study found higher levels of 90-day readmission, dissatisfaction, and burden for healthcare providers among patients with depression [33]. Another study showed that patients with preoperative anxiety and pain catastrophizing had more postoperative pain, poorer preoperative and postoperative knee function, and a higher dissatisfaction rate; the authors recommended the preoperative screening and treatment of psychological factors [30].

One possible interpretation of the above-mentioned studies is that patients with anxiety and depression would not benefit from TKA surgery, and, hence, would be difficult for knee surgeons and other healthcare providers to handle postoperatively, given their repeated complaints of dissatisfaction. This might lead to the exclusion of patients who need to undergo TKA, potentially unnecessarily increasing patient suffering and paradoxically increasing the dissatisfaction rate. A global effort is now being made to prevent the increasing rate of dissatisfaction that might be expected due to the increasing number of TKA surgeries performed worldwide $[1,2,11]$.

Despite the large number of studies investigating dissatisfied patients after TKA, little attention has been paid to the actual benefits of TKA surgery in patients with preoperative anxiety and depression. Our aim in this cohort study was, therefore, to investigate the prevalence of symptom improvement among patients with preoperative anxiety and/ or depression in comparison with patients who did not have anxiety and/or depression. This might improve our understanding and evaluation of patients in whom knee complaints coexist with preoperative psychological distress; this might in turn decrease the unnecessary delay of TKA surgery.

\section{Method}

\section{Patient selection}

This was a prospective cohort study of a consecutive sample of Swedish patients who underwent TKA between April 2016 and July 2018. The patients received their primary total knee prosthesis at three hospitals in Mid-Sweden. All the orthopedic surgeons used a standard paramedian approach with one of three types of knee prosthesis: Genesis II (Smith \& Nephew, Watford, UK), NexGen MBT (Zimmer-Biomet, Warsaw, IN, USA), and Journey TKA (Smith \& Nephew, London, UK).

Inclusion criterion: all patients with knee arthritis (for primary osteoarthritis, secondary osteoarthritis, and inflammatory arthritis) who were scheduled for primary TKA.

Exclusion criteria: any patients having revision arthroplasty, medial unicompartmental arthroplasty, lateral unicompartmental arthroplasty, and patella-femoral arthroplasty.

All patients were asked to complete a questionnaire including patient-reported outcome measures (PROMs), regardless of whether or not they were included in the study. PROMs data are routinely collected before and one year after TKA for the Swedish Knee Arthroplasty Registry (SKAR) at the clinics where the study was conducted. These data were expected to cover around 500 patients during the study period (2016-2018), which was considered a representative sample. It was decided to include the first 500 consecutive patients with complete data.

SKAR was set up in 1975 and is now one of the most trusted such registries in the world [12]. It uses the individual-based registration of patients and TKA surgery. SKAR provided us with the PROMs questionnaire used annually by the registry, created software for entering and calculating the HADS score, and gave us access to its data program for entering PROMs, including HADS data. This access concerned only our patients and was restricted to the common database. We, therefore, took advantage of SKAR routines in terms of using a validated questionnaire and high-quality software that protects patient identity.

Demographic data for the patients included in the study are given in Table 1.

\section{Outcome measures}

A psychometrically validated questionnaire was used to collect the data [36-38]. It was completed by the patients at two time points: before surgery and one year afterwards. This questionnaire included four different measures of PROMs: the Knee Injury and Osteoarthritis Outcome Score (KOOS), which consists of five subscales; general health state rated on a scale of 0-100; a visual analog scale (VAS) for rating pain intensity from 0 to 100 ; and a five-dimensional general quality of life instrument (EQ-5D-3L).

The questionnaire also included the Hospital Anxiety and Depression Scale (HADS), which consists of seven items for anxiety and seven items for depression. Each item has four possible answers, i.e., 0-3. HADS has a minimum score of 0 and a maximum of 21 for each dimension. HADS is a screening instrument designed mainly to 
Table 1 Demographics of the TKA patients included in the study $(n=458)$

\begin{tabular}{|c|c|c|c|c|c|c|}
\hline Variables & $\mathrm{PA}(n=70)$ & NPA $(n=388)$ & $P$-value & $\mathrm{PD}(n=44)$ & $\operatorname{NPD}(n=414)$ & $P$-value* \\
\hline \multicolumn{7}{|l|}{ Gender, $n(\%)$} \\
\hline Female & $43(61)$ & $196(51)$ & \multirow[t]{2}{*}{0.09} & $29(66)$ & $210(51)$ & \multirow[t]{2}{*}{0.05} \\
\hline Male & $27(39)$ & $192(49)$ & & $15(34)$ & $204(49)$ & \\
\hline Age, $m$ & 68 & 70 & 0.01 & 66 & 70 & 0.003 \\
\hline BMI & 30 & 29 & 0.08 & 31 & 29 & 0.001 \\
\hline \multicolumn{7}{|l|}{ ASA class } \\
\hline I & $18(26)$ & $99(25)$ & \multirow[t]{3}{*}{0.94} & $15(34)$ & $102(25)$ & \multirow[t]{3}{*}{0.27} \\
\hline II & $45(64)$ & $255(66)$ & & $24(55)$ & $276(66)$ & \\
\hline III & $7(10)$ & $34(9)$ & & $5(11)$ & $36(9)$ & \\
\hline \multicolumn{7}{|l|}{ Charnley class } \\
\hline A & $14(20)$ & $98(25)$ & \multirow[t]{5}{*}{0.37} & $7(16)$ & $105(25)$ & \multirow[t]{5}{*}{0.21} \\
\hline B1 & $16(23)$ & $97(25)$ & & $8(18)$ & $105(25)$ & \\
\hline B2 & $7(10)$ & $58(15)$ & & $6(14)$ & $59(14)$ & \\
\hline $\mathrm{C}$ & $32(46)$ & $131(34)$ & & $22(50)$ & $141(34)$ & \\
\hline Missing & $1(1)$ & $4(1)$ & & $1(2)$ & $4(1)$ & \\
\hline \multicolumn{7}{|l|}{ Diagnosis, $n(\%)$} \\
\hline Osteoarthrosis & $65(93)$ & $377(97)$ & \multirow[t]{4}{*}{0.29} & $42(96)$ & $400(97)$ & \multirow[t]{4}{*}{0.61} \\
\hline Osteonecrosis & $2(3)$ & $5(1)$ & & $1(2)$ & $6(1)$ & \\
\hline Rheumatoid arthritis & $2(3)$ & $3(1)$ & & $0(0)$ & $5(1)$ & \\
\hline Fracture sequelae & $1(1)$ & $3(1)$ & & $1(2)$ & $3(1)$ & \\
\hline \multicolumn{7}{|c|}{ OARSI-Responder, $n(\%)$} \\
\hline Non-responder & $12(17)$ & $38(10)$ & \multirow[t]{2}{*}{0.15} & $4(9)$ & $46(11)$ & \multirow[t]{2}{*}{0.39} \\
\hline Responder & $58(83)$ & $346(90)$ & & $40(91)$ & $364(89)$ & \\
\hline Patella component & $0(0)$ & $9(2)$ & 0.19 & $1(2)$ & $8(2)$ & 0.87 \\
\hline \multicolumn{7}{|l|}{ Previous surgery } \\
\hline No & $57(81)$ & $302(78)$ & \multirow[t]{2}{*}{0.34} & $34(77)$ & $325(80)$ & \multirow[t]{2}{*}{0.63} \\
\hline Yes & $13(19)$ & $86(22)$ & & $10(23)$ & $89(20)$ & \\
\hline \multicolumn{7}{|l|}{ Anesthesia, $n(\%)$} \\
\hline General & $40(57)$ & $221(57)$ & \multirow[t]{2}{*}{0.97} & $21(48)$ & $240(58)$ & \multirow[t]{2}{*}{0.19} \\
\hline Spinal & $30(43)$ & $167(43)$ & & $23(52)$ & $174(42)$ & \\
\hline \multicolumn{7}{|l|}{ LIA, $n(\%)$} \\
\hline No & $1(1)$ & $5(2)$ & \multirow[t]{2}{*}{0.83} & $1(2)$ & $407(98)$ & \multirow[t]{2}{*}{0.75} \\
\hline Yes & $69(99)$ & $388(98)$ & & $43(98)$ & $7(2)$ & \\
\hline \multicolumn{7}{|l|}{ Tourniquet, $n(\%)$} \\
\hline No & $41(59)$ & $210(54)$ & 0.49 & $19(43)$ & $232(56)$ & 0.10 \\
\hline Yes & $29(41)$ & $178(46)$ & & $25(57)$ & $182(44)$ & \\
\hline Surgical time, $\min$ & 89 & 87 & 0.51 & 85 & 88 & 0.33 \\
\hline Side, $n(\%)$ & & & & & & \\
\hline Right knee & $42(60)$ & $193(50)$ & 0.11 & $26(59)$ & $209(51)$ & 0.27 \\
\hline Left knee & $28(40)$ & $195(50)$ & & $18(41)$ & 205 (49) & \\
\hline
\end{tabular}

$B M I$ body mass index, $P A$ preoperative anxiety, $N P A$ no preoperative anxiety, $P D$ preoperative depression, $N P D$ no preoperative depression, $n$ number, \% percent, $m$ mean, ASA American Society of Anesthesiologists, OARSI Osteoarthritis Research Society International, LIA local infiltration anesthesia

*Chi-square test for binary data and independent t-test for continuous data explore anxiety and depression in patients with somatic and psychotic disorders [39]. It takes 2-5 min to complete and has been translated into many languages $[32,40]$. It has good validity in defining anxiety and depression and in assessing their severity. It has been shown to be sensitive to change both during the disease course and in response to interventions [38-40]. 
We first categorized the patients, based on their HADS scores, into two groups: one with anxiety and another without anxiety; similarly, we obtained two groups with and

Table 2 Demographics of the patients with incomplete data compared with complete data

\begin{tabular}{llll}
\hline Characteristics & $\begin{array}{l}\text { Incomplete data, } \\
n \text { (total } n=157)\end{array}$ & $\begin{array}{l}\text { Complete data, } \\
n \text { (total=458) }\end{array}$ & $P$-value* \\
\hline Gender, $n(\%)$ & & & 0.07 \\
Female & $95(60)$ & $239(52)$ & \\
Male & $62(40)$ & $219(48)$ & 0.06 \\
Age, $m$ & 68 & 70 & 0.003 \\
BMI, $m$ & 30 & 29 & 0.07 \\
ASA, $n(\%)$ & & $117(25)$ & \\
I & $26(16)$ & $300(66)$ & \\
II & $115(73)$ & $41(9)$ & \\
III & $16(10)$ & & \\
Charnley class, $n(\%)$ & & $112(25)$ & \\
A & $15(9)$ & $113(25)$ & \\
B1 & $17(11)$ & $65(14)$ & \\
B2 & $17(11)$ & $163(35)$ & \\
C & $53(34)$ & $5(1)$ & \\
Missing & $55(35)$ & & \\
PAS, $n(\%)$ & & $388(85)$ & \\
No & $68(72)$ & $70(15)$ & \\
Yes & $26(27)$ & & \\
Missing & 63 & & \\
PDS, $n(\%)$ & $81(87)$ & & \\
No & $12(13)$ & & \\
Yes & 64 & & \\
Missing & & & \\
\hline
\end{tabular}

$B M I$ body mass index, ASA American Society of Anesthesiologists, $P A S$ preoperative anxiety symptoms, $P D S$ preoperative depression symptoms

*Chi-square test for binary data and independent t-test for continuous data without depression symptoms. Patients were regarded as having anxiety and/or depression symptoms if their relevant HADS score was more than seven [38].

Preoperative VAS expectations were assessed on a $0-100$ scale, with 0 representing no pain and 100 the worst experienced pain. Postoperative VAS satisfaction was assessed using a similar $0-100$ scale; the scale was completed by the patients one year after TKA and indicated the degree of satisfaction with their knee pain. "How good is your knee?" was another 0-100 scale (best-worst) used to assess postoperative patient satisfaction after one year [12].

\section{Analysis}

Chi-square testing was used to compare binary data, while independent $t$-testing was used to compare continuous data for demographic variables, expectations, and satisfaction (Tables 1, 2, and 7).

A paired $t$ test was used to compare changes in mean differences in every group before surgery and one year after surgery regarding the outcome measures (i.e., EQ-5D-3L, VAS pain, general health state, and the KOOS subscales). We divided the patients into four groups (i.e., with preoperative anxiety, without preoperative anxiety, with preoperative depression, and without preoperative depression) and used a paired Student's $t$-test to compare the mean differences in outcome measures in all four groups before and one year after TKA.

Two-way repeated-measures ANOVA was used to compare the mean differences within each group (i.e., anxiety, no anxiety, depression, and no depression) and to compare the mean differences between groups (i.e., anxiety vs. no anxiety and depression vs. no depression) (Tables 3 and 4).

A Chi-square test was used to compare the proportions of patients with and without preoperative anxiety in relation to clinical improvement in EQ-5D-3L, VAS pain, general
Table 3 Change in outcome variables one year after TKA in patient groups without and with preoperative anxiety symptom

\begin{tabular}{|c|c|c|c|c|c|c|}
\hline \multirow[t]{2}{*}{ Outcome variable } & \multicolumn{2}{|c|}{$\begin{array}{l}\text { No preoperative anxiety } \\
(n=388)\end{array}$} & \multicolumn{2}{|c|}{$\begin{array}{l}\text { Preoperative anxiety } \\
(n=70)\end{array}$} & \multirow[t]{2}{*}{$\begin{array}{l}\text { MD between } \\
\text { groups }\end{array}$} & \multirow[t]{2}{*}{ Sig. ${ }^{* *}$} \\
\hline & MD & Sig (2-tailed) ${ }^{*}$ & $\mathrm{MD}$ & Sig (2-tailed) & & \\
\hline EQ-5D-3L & 0.32 & $<0.001$ & 0.33 & $<0.001$ & 0.01 & 0.89 \\
\hline VAS pain & -48 & $<0.001$ & -45 & $<0.001$ & 3 & 0.35 \\
\hline General health state & 11 & $<0.001$ & 16 & $<0.001$ & 5 & 0.17 \\
\hline KOOS-symptoms & 30.6 & $<0.001$ & 30 & $<0.001$ & 0.6 & 0.83 \\
\hline KOOS-pain & 42 & $<0.001$ & 37.2 & $<0.001$ & 4.8 & 0.08 \\
\hline KOOS-ADL & 33 & $<0.001$ & 30 & $<0.001$ & 3 & 0.18 \\
\hline KOOS-sport & 28 & $<0.001$ & 18 & $<0.001$ & 9 & 0.006 \\
\hline KOOS-QoL & 43 & $<0.001$ & 38 & $<0.001$ & 5 & 0.08 \\
\hline
\end{tabular}

$M D$ mean difference, $V A S$ visual analog scale, KOOS Knee Injury and Osteoarthritis Outcome Score, $A D L$ activities of daily living, $Q o L$ quality of life

*Paired $t$-test; ** Two-way repeated-measures ANOVA 
Table 4 Change in outcome variables one year after TKA in patient groups without and with preoperative depression symptoms

\begin{tabular}{|c|c|c|c|c|c|c|}
\hline \multirow[t]{2}{*}{ Outcome variable } & \multicolumn{2}{|c|}{$\begin{array}{l}\text { No preoperative depression } \\
(n=414)\end{array}$} & \multicolumn{3}{|c|}{ Preoperative depression $(n=44)$} & \multirow[t]{2}{*}{ Sig.** } \\
\hline & MD & Sig (2-tailed)* & MD & Sig (2-tailed) & $\begin{array}{l}\text { MD between } \\
\text { groups }\end{array}$ & \\
\hline EQ-5D-3L & 0.32 & $<0.001$ & 0.33 & $<0.001$ & 0.01 & 0.86 \\
\hline VAS pain & -48 & $<0.001$ & -49 & $<0.001$ & 1 & 0.78 \\
\hline General health state & 11.5 & $<0.001$ & 14 & $<0.001$ & 2.5 & 0.54 \\
\hline KOOS-symptoms & 30.5 & $<0.001$ & 31 & $<0.001$ & 0.5 & 0.89 \\
\hline KOOS-pain & 41 & $<0.001$ & 40 & $<0.001$ & 1 & 0.80 \\
\hline KOOS-ADL & 33 & $<0.001$ & 30 & $<0.001$ & 3 & 0.40 \\
\hline KOOS-sport & 27 & $<0.001$ & 18 & $<0.001$ & 9 & 0.03 \\
\hline KOOS-QoL & 43 & $<0.001$ & 37 & $<0.001$ & 6 & 0.15 \\
\hline
\end{tabular}

$M D$ mean difference, VAS visual analog scale, KOOS Knee Injury and Osteoarthritis Outcome Score, $A D L$ activities of daily living, $Q o L$ quality of life

*Paired $t$-test; ** Two-way repeated-measures ANOVA health state, and each KOOS subscale. A $P$-value of less than 0.05 was regarded as statistically significant. To determine whether the improvement of an outcome measure was clinically significant, specific cut-off points were used as follows: 0.25 for EQ-5D-3L, 15 for VAS pain, 15 for the general health state variable, and eight for each KOOS subscale $[12,36]$. Similar comparisons were performed for patients with and without preoperative depression symptoms. Thus, cross tables were used to compare two categories of preoperative psychological state (i.e., with anxiety/depression and without anxiety/depression) in relation to the two categories of 1-year postoperative outcome measures (i.e., clinically significant improvement and non-clinically significant improvement) (Tables 5 and 6).

VAS expectations were sorted into three categories: high expectations (0-30), moderate expectations (30-60), and low expectations (60-100). Similar categories were formed for VAS satisfaction, i.e., high/very high satisfaction, moderate satisfaction, and low/very low satisfaction. Similarly, we categorized the "How good is your knee?" variable as good/ very good knee, moderately good knee, and bad/very bad knee. The mean values for VAS expectations, VAS satisfaction, and "How good is your knee?" were also calculated (Table 7) [12]. SPSS, version 25 (IBM, SPSS Inc., Chicago, Illinois, USA) was used for the data analysis.

\section{Results}

Between April 2016 and July 2018, 719 patients from MidSweden underwent knee arthroplasty. The participation response rate was $91 \%$ (65 patients declined). After excluding patients who declined $(n=65)$, those with incomplete data $(n=157)$, and those who did not meet the inclusion
Table 5 Comparison between patient groups with and without preoperative anxiety symptoms in relation to postoperative improvement in outcome measures

\begin{tabular}{|c|c|c|c|c|}
\hline Variable & PA $n(\%)$ & NPA $n(\%)$ & OR & $P$-value ${ }^{*}$ \\
\hline \multicolumn{5}{|c|}{ EQ-5D-3L } \\
\hline CSI & $37(53)$ & $222(58)$ & 1.08 & 0.46 \\
\hline No CSI & $33(47)$ & $164(42)$ & & \\
\hline \multicolumn{5}{|l|}{ VAS pain } \\
\hline CSI & $68(97)$ & $374(98)$ & 0.82 & 0.80 \\
\hline No CSI & $2(3)$ & $9(2)$ & & \\
\hline \multicolumn{5}{|c|}{ General health state } \\
\hline CSI & $38(55)$ & $138(38)$ & 1.41 & 0.004 \\
\hline No CSI & $31(45)$ & $240(62)$ & & \\
\hline \multicolumn{5}{|c|}{ KOOS-symptoms } \\
\hline CSI & $56(80)$ & $329(85)$ & 1.06 & 0.31 \\
\hline No CSI & $14(20)$ & $59(15)$ & & \\
\hline \multicolumn{5}{|c|}{ KOOS-pain } \\
\hline CSI & $62(89)$ & $365(95)$ & 1.07 & 0.03 \\
\hline No CSI & $8(11)$ & $19(5)$ & & \\
\hline \multicolumn{5}{|c|}{ KOOS-ADL } \\
\hline CSI & $59(84)$ & $351(91)$ & 1.07 & 0.08 \\
\hline No CSI & $11(16)$ & $35(9)$ & & \\
\hline \multicolumn{5}{|c|}{ KOOS-sport } \\
\hline CSI & $43(63)$ & $267(72)$ & 1.14 & 0.12 \\
\hline No CSI & $25(37)$ & $102(28)$ & & \\
\hline \multicolumn{5}{|c|}{ KOOS-QoL } \\
\hline CSI & $65(93)$ & $355(92)$ & 0.99 & 0.85 \\
\hline No CSI & $5(7)$ & $30(8)$ & & \\
\hline
\end{tabular}

$P A$ preoperative anxiety, $N P A$ no preoperative anxiety, $n(\%)$ number (percent), OR odds ratio, $P$-value probability value, $C S I$ clinically significant improvement

*Chi-square test 
Table 6 Comparison between patient groups with and without preoperative depression symptom in relation to postoperative improvement in outcome measures

\begin{tabular}{|c|c|c|c|c|}
\hline Variable & $\mathrm{PD} n(\%)$ & NPD $n(\%)$ & OR & $P$-value* \\
\hline \multicolumn{5}{|c|}{ EQ-5D-3L } \\
\hline CSI & $22(51)$ & $237(57)$ & 1.12 & 0.43 \\
\hline No CSI & $21(49)$ & $176(43)$ & & \\
\hline \multicolumn{5}{|l|}{ VAS pain } \\
\hline CSI & $41(95)$ & $401(98)$ & 1.02 & 0.32 \\
\hline No CSI & $2(5)$ & $9(2)$ & & \\
\hline \multicolumn{5}{|c|}{ General health state } \\
\hline CSI & $23(52)$ & $153(38)$ & 1.33 & 0.04 \\
\hline No CSI & $21(48)$ & $250(62)$ & & \\
\hline \multicolumn{5}{|c|}{ KOOS-symptoms } \\
\hline CSI & $41(93)$ & $386(94)$ & 1.01 & 0.79 \\
\hline No CSI & $3(7)$ & $24(6)$ & & \\
\hline \multicolumn{5}{|c|}{ KOOS-pain } \\
\hline CSI & $42(93)$ & $386(94)$ & 1.01 & 0.79 \\
\hline No CSI & $3(7)$ & $24(6)$ & & \\
\hline \multicolumn{5}{|c|}{ KOOS-ADL } \\
\hline CSI & 39(89) & $371(90)$ & 1.01 & 0.76 \\
\hline No CSI & $5(11)$ & $41(10)$ & & \\
\hline \multicolumn{5}{|c|}{ KOOS-sport } \\
\hline CSI & $30(68)$ & $280(71)$ & 1.04 & 0.67 \\
\hline No CSI & $14(32)$ & 113(29) & & \\
\hline \multicolumn{5}{|c|}{ KOOS-QoL } \\
\hline CSI & $39(91)$ & $381(93)$ & 1.02 & 0.76 \\
\hline No CSI & $4(9)$ & $31(7)$ & & \\
\hline
\end{tabular}

$P D$ preoperative depression, NPA no preoperative depression, $n(\%)$ number (percent), $O R$ odds ratio, $P$-value probability value, $C S I$ clinically significant improvement

*Chi-square test

criteria $(n=39)$, a total of 458 patients with complete data were included in the study (Fig. 1).

Missing data were distributed randomly and did not affect any specific group or hospital. The demographics of the patients with missing data are shown in Table 2. Patients with incomplete data had significantly higher body mass index (BMI; $P=0.003$ ), a lower percentage of Charnley class $\mathrm{A}$ and $\mathrm{B}(P<0.001)$, and probably more preoperative anxiety $(P=0.004)$. There were no statistically significant differences in other variables, as shown in Table 2.

We first categorized the data according to the preoperative anxiety and depression state: 70 patients $(15.3 \%)$ had preoperative anxiety symptoms and the remaining 388 $(84.7 \%)$ did not have anxiety, while 44 patients $(9.6 \%)$ had preoperative depression symptoms and the remaining 414 (90.4\%) did not have depression.

Demographic data analysis revealed no statistically significant differences between the groups with and without preoperative anxiety and/or depression (Table 1), except that patients with preoperative anxiety and/or depression were younger than those without anxiety and/or depression $(P=0.01$ and 0.003 , respectively). The mean BMI in the group with preoperative depression was significantly higher than in the group without preoperative depression (31 vs. 29, $P=0.001)$.

Comparisons were performed for each group before and one year after surgery. All groups displayed statistically and clinically significant improvement in all outcome measures (Tables 3 and 4): EQ-5D-3L, VAS pain, general health status, and all KOOS subscales (i.e., symptoms, pain, activities of daily living, sport, and quality of life; $P<0.001)$.

Patients with preoperative anxiety and/or depression generally displayed less improvement in the outcome measures than did the groups without anxiety and/or depression, but most of the differences were not statistically significant. One exception was the mean difference in KOOS-sport, which was significantly higher in the non-anxiety and nondepression groups than in the anxiety and depression groups; moreover, two of the further three significant results were in favor of the anxiety and/or depression groups (Tables 5 and 6). The only statistically significant differences were in clinically significant improvements of KOOS-pain in $95 \%$ of patients without preoperative anxiety versus $89 \%$ of patients with preoperative anxiety $(P=0.03)$, of the general health state in $55 \%$ of patients with preoperative anxiety versus $38 \%$ of patients without preoperative anxiety $(P=0.004)$, and of general health state in $52 \%$ of patients with preoperative depression versus $38 \%$ of patients without preoperative depression $(P=0.04)$ (Tables 5 and 6).

Our data indicated that $92 \%$ of patients had high preoperative VAS expectations, $81 \%$ high/very high VAS satisfaction, and $70 \%$ good/very good knee one year after TKA. Patients without preoperative anxiety and/or depression had slightly higher values for the preoperative VAS expectations, VAS satisfaction, and "How good is your knee?" variables. The differences among either the proportions or mean values were not statistically significant, however, except in the "How good is your knee?" variable, for which the mean value in the non-anxiety group was statistically significantly higher than in the anxiety group (23 vs. 29 , $P=0.03$ ) (Table 7).

\section{Discussion}

This study examined clinical improvements one year after total knee arthroplasty in patients with knee arthritis. The results indicate significant improvements in outcome measures regardless of the patients' presurgical anxiety and/or depression symptoms. Although patients with presurgical anxiety and/or depression symptoms generally 
Table 7 Patients' preoperative expectations and postoperative satisfaction $(n=458)$

\begin{tabular}{|c|c|c|c|c|c|c|}
\hline Variables & $\mathrm{PA}(n=70)$ & NPA $(n=388)$ & $P$-value* & $\mathrm{PD}(n=44)$ & $\mathrm{NPD}(n=414)$ & $P$-value* \\
\hline \multicolumn{7}{|l|}{ VAS expectations, $n(\%)$} \\
\hline High expectations & $66(92)$ & $455(92)$ & 0.99 & $41(93)$ & $379(92)$ & 0.71 \\
\hline Moderate expectations & $4(7)$ & $28(7)$ & & $3(7)$ & $29(7)$ & \\
\hline Low expectations & $1(1)$ & $5(1)$ & & $0(0)$ & $6(1)$ & \\
\hline VAS expectations, $m$ & 18.45 & 16.65 & 0.28 & 16.13 & 17 & 0.66 \\
\hline \multicolumn{7}{|l|}{ VAS satisfaction, $n(\%)$} \\
\hline High/very high satisfaction & $56(78)$ & $315(81)$ & 0.41 & $35(80)$ & $337(80)$ & 0.54 \\
\hline Moderate satisfaction & $5(8)$ & $37(10)$ & & $3(7)$ & $40(10)$ & \\
\hline Low/very low satisfaction & $9(13)$ & $32(9)$ & & $6(13)$ & $37(9)$ & \\
\hline VAS satisfaction, m & 22 & 17 & 0.12 & 21 & 17 & 0.30 \\
\hline \multicolumn{7}{|l|}{ "How good is your knee?", $n(\%)$} \\
\hline Good/very good knee & $46(65)$ & $274(70)$ & 0.62 & $28(64)$ & $292(70)$ & 0.73 \\
\hline Moderately good knee & $17(25)$ & $87(22)$ & & $12(27)$ & $92(22)$ & \\
\hline $\mathrm{Bad} /$ very bad knee & $7(10)$ & $27(7)$ & & $4(9)$ & $30(7)$ & \\
\hline "How good is your knee?", $\mathrm{m}$ & 29 & 23 & 0.03 & 28.53 & 23.68 & 0.18 \\
\hline
\end{tabular}

$V A S$ visual analog scale, $P A$ preoperative anxiety, $N P A$ no preoperative anxiety, $P D$ preoperative depression, $N P D$ no preoperative depression, $n$ number, $\%$ percent, $m$ mean

*Chi-square test for binary data and independent $t$-test for continuous data

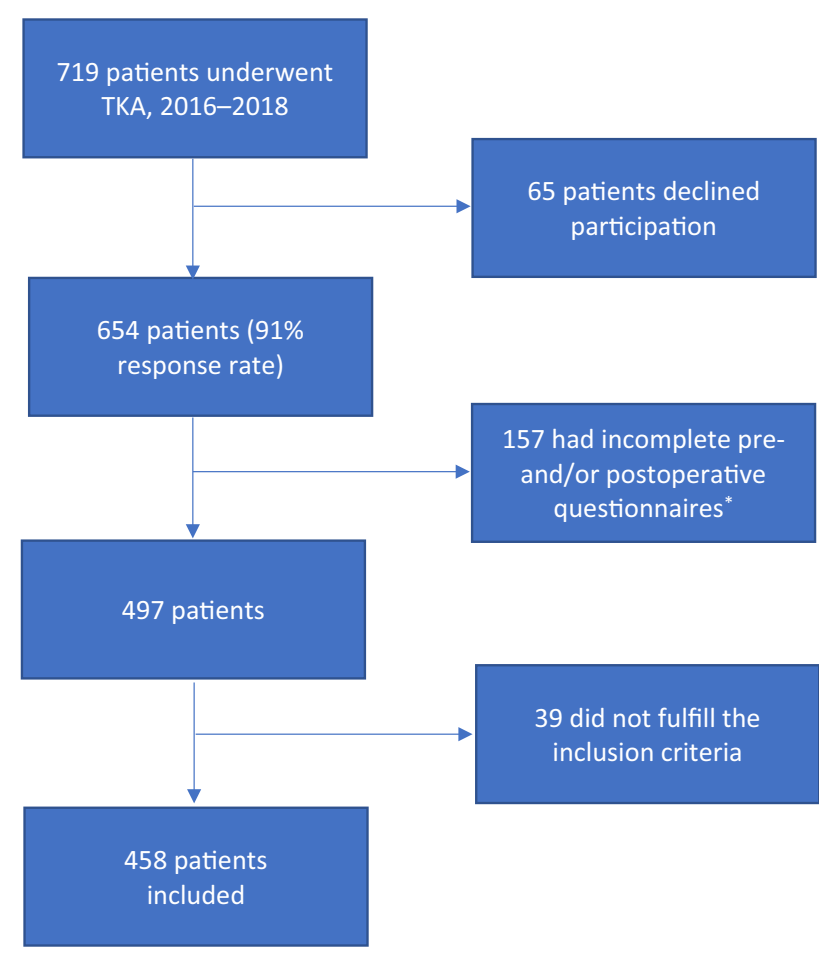

Fig. 1 Flow chart of the patients included in the study. *Reasons for missing data were: (1)At the beginning of the study the nurses had not yet got into the routine of distributing the questionnaire, which caused them to miss some patients; (2)Patients delaying their answers for more than 3 months; (3) Incomplete answering of the questionnaire; (4) Patients not returning the preoperative or postoperative questionnaire despite reminders; (5) Death displayed less improvement than did patients without these symptoms, the differences between the groups were not statistically significant in this cohort except in the case of pain and sport/recreational activities.

Many studies have shown that TKA is an effective procedure for improving pain and function in patients with knee arthritis $[1,4,15,16]$. However, there is an ongoing worldwide effort to further improve the outcome after TKA, because of the expected increase in the number of patients undergoing TKA, which entails a risk of increasing the dissatisfaction rate after surgery [1]. Our study is in agreement with the literature regarding the improvement of both generic and knee-specific outcome measures one year after TKA. The highest improvement was seen in VAS pain and KOOS-QoL (i.e., quality of life), regardless of presurgical psychological state.

In the past 7 years, more attention has been paid to the negative effects of presurgical psychological distress on patient satisfaction after TKA [13, 14, 29-32], leading to the improved presurgical assessment of patients and improved patient selection for surgery. One study revealed preoperative anxiety and/or depression to be an important factor predicting patient satisfaction after TKA [14]. The authors highlighted that patients with preoperative anxiety and/or depression had six times more risk of being dissatisfied than did patients without these symptoms; this study and other studies recommended preoperative psychological assessment to possibly decrease the dissatisfaction rate [14]. Another systemic review also focused on preoperative psychological distress (i.e., psychological 
anxiety and/or depression) as a predictor of poor outcome after TKA [13].

Many of the above-mentioned studies strove to identify patients with psychological illness, which might be misinterpreted by orthopedic surgeons as indicating that they should not operate on patients with preoperative psychological illness. This delay of surgery in patients with presurgical psychological distress has not yet been well studied in the literature, but it is not uncommon in clinical practice. Our study, however, highlights that TKA is also effective in patients with preoperative anxiety and/or depression symptoms, as these patients experienced statistically and clinically significant improvement in both generic and knee-specific outcome measures. The present results can help to increase awareness and accurate assessment of this category of patients, which might in turn prevent unnecessary delays of surgery and hence improve these patients' quality of life.

Although patients with presurgical anxiety and/or depression displayed less improvement than did patients without such symptoms, the within-group improvement was still considered large and both clinically and statistically significant. Moreover, the mean differences between the groups with and without anxiety and/or depression symptoms were not statistically significant, except in the case of sport and recreational activities. Further studies with larger sample sizes might be able to uncover statistically significant differences between the groups, though these will not necessarily be clinically important. Another exception to the above was that a significantly higher proportion of patients without presurgical anxiety saw an improvement in KOOS-pain than did those with presurgical anxiety symptoms.

Another interesting result concerned the patients' general health state: a significantly higher proportion of patients with preoperative anxiety and/or depression displayed improvement in this measure than did those without such symptoms. This might offer further evidence of the effectiveness of TKA in improving general quality of life, reducing pain, and improving knee function in patients with preoperative anxiety and/or depression symptoms. The SKAR annual report showed an improvement in the general health state of TKA patients one year after the performance of surgery [12], though there was no stratification of the groups based on patients psychological distress (anxiety/depression symptoms).

Patients' preoperative expectations in relation to postoperative satisfaction have also been studied [6, 8, 25-28], showing that fulfillment of patients' expectations predicted postoperative patient satisfaction after TKA surgery. In a qualitative study, we found that patients' expectations were still the major contributing factor affecting patient contentment one year after surgery [25].

In this study, the differences between patients with and without preoperative anxiety and/or depression symptoms were not statistically significant. These results provide further evidence that patients with preoperative anxiety and/ or depression symptoms have as high preoperative expectations and postoperative satisfaction as do patients without these symptoms. Increasing the size of the patient sample in future studies might reveal statistically significant differences. These differences should not lead to discrimination against patients at a group level, but rather should help TKA surgeons improve their assessment of patients at the individual level.

\section{Strengths and weaknesses}

\begin{tabular}{|c|c|c|c|}
\hline$n$ & Limitations & $n$ & Strength \\
\hline 1 & $\begin{array}{l}\text { Insufficient data for between-group } \\
\text { comparison }\end{array}$ & 1 & $\begin{array}{l}\text { Sufficient data } \\
\text { for within- } \\
\text { group com- } \\
\text { parison }\end{array}$ \\
\hline 2 & No objective physical knee tests & 2 & $\begin{array}{l}\text { Consecutive } \\
\text { prospective } \\
\text { sample }\end{array}$ \\
\hline
\end{tabular}

We obtained complete data from 458 patients, which was sufficient to yield clinically and statistically significant results regarding within-group comparisons. However, this patient sample was insufficient to yield statistically significant results regarding the comparison of patients with versus without anxiety and/or depression in relation to clinical improvement in the KOOS subscales.

Another weakness of this study was that improvement was considered only on the basis of PROMs. We did not administer any objective physical tests such as walking tests or stair-climbing tests, and we did not directly ask the patients whether they were really satisfied with the surgery one year postoperatively.

A strength of this study was its prospective cohort design, including a consecutive group of knee arthritis patients who underwent surgery during 2016-2018. This helped minimize the selection bias, even though $24 \%$ (157/654) of cases were lost.

\section{Conclusions}

TKA produces excellent subjective improvement in patients' quality of life, pain, activities of daily living, knee symptoms, recreational activities, and general health state. Patients with presurgical anxiety and/or depression symptoms should be accurately assessed before surgery; however, 
they benefit greatly from surgery and should not be discriminated against based on presurgical psychological distress.

Acknowledgements Open access funding provided by Örebro University. We thank Ole Brus (Clinical Epidemiology and Biostatistics, School of Medical Sciences, Örebro University, Örebro, Sweden) for statistical advice and help with the SPSS software package, and Inger Andersson (nurse and Chief Coordinator, Department of Orthopedics, Örebro University Hospital, Örebro, Sweden) for her well-structured and organized data entry and for sending reminders to the patients who did not respond in good time.

Authors' contributions AM created the study protocol and design, collected the data, performed the analysis, and wrote the manuscript. MHN contributed to the study design, the analysis, and the revision of the manuscript. PW contributed to the study design, the data collection, and the revision of the manuscript.

\section{Compliance with ethical standards}

Conflict of interest The authors declare no conflict of interest.

Ethical approval The study was approved by the Regional Ethical Review Board of Uppsala, Sweden (ref: 2016/191).

Informed consent All participants gave their verbal and written consent before surgery, and a new written consent form was sent together with the questionnaire one year after surgery. The data were treated with full confidentiality.

Open Access This article is licensed under a Creative Commons Attribution 4.0 International License, which permits use, sharing, adaptation, distribution and reproduction in any medium or format, as long as you give appropriate credit to the original author(s) and the source, provide a link to the Creative Commons licence, and indicate if changes were made. The images or other third party material in this article are included in the article's Creative Commons licence, unless indicated otherwise in a credit line to the material. If material is not included in the article's Creative Commons licence and your intended use is not permitted by statutory regulation or exceeds the permitted use, you will need to obtain permission directly from the copyright holder. To view a copy of this licence, visit http://creativecommons.org/licenses/by/4.0/.

\section{References}

1. Carr AJ et al (2012) Knee replacement. Lancet 379(9823):1331-1340

2. Giwnewer U et al (2016) Treatment for osteoarthritis of the knee. Harefuah 155(7):403-406

3. Rubin LE, Blood TD, Defillo-Draiby JC (2016) Total hip and knee arthroplasty in patients older than age 80 years. J Am Acad Orthop Surg 24(10):683-690

4. Austin DC et al (2018) Patient outcomes after total knee arthroplasty in patients older than 80 years. J Arthroplast 33(11):3465-3473

5. Wylde V et al (2019) Variability in long-term pain and function trajectories after total knee replacement: a cohort study. Orthop Traumatol Surg Res 105(7):1345-1350

6. Ghomrawi HMK et al (2019) Preoperative expectations associated with postoperative dissatisfaction after total knee arthroplasty: a cohort study. J Am Acad Orthop Surg. https://doi.org/10.5435/ JAAOS-D-18-00785

7. Dhurve $\mathrm{K}$ et al (2017) Multifactorial analysis of dissatisfaction after primary total knee replacement. Knee 24(4):856-862

8. Deakin AH et al (2019) Fulfilment of preoperative expectations and postoperative patient satisfaction after total knee replacement. A prospective analysis of 200 patients. Knee 26(6):1403-1412

9. Khatib Y et al (2020) Patient satisfaction and perception of success after total knee arthroplasty are more strongly associated with patient factors and complications than surgical or anaesthetic factors. Knee Surg Sports Traumatol Arthrosc. https://doi. org/10.1007/s00167-019-05804-9

10. Sauder N et al (2020) Regional differences between the US, Scandinavia, and South Korea in patient demographics and patientreported outcomes for primary total knee arthroplasty. Arch Orthop Trauma Surg 140(1):93-108

11. Hamilton DF et al (2015) Dealing with the predicted increase in demand for revision total knee arthroplasty: challenges, risks and opportunities. Bone Joint J 97(6):723-728

12. SKAR, Annual report Swedish knee arthroplasty register 2018, SKAR, Editor. 2019: Sweden

13. Alattas SA et al (2017) Greater pre-operative anxiety, pain and poorer function predict a worse outcome of a total knee arthroplasty. Knee Surg Sports Traumatol Arthrosc 25(11):3403-3410

14. Ali A et al (2017) Preoperative anxiety and depression correlate with dissatisfaction after total knee arthroplasty: a prospective longitudinal cohort study of 186 patients, with 4-year follow-up. J Arthroplasty 32(3):767-770

15. Bontempi M et al (2017) Total knee replacement: intraoperative and postoperative kinematic assessment. Acta Biomed $88(2$ s $): 32-37$

16. Gibon E, Goodman MJ, Goodman SB (2017) Patient satisfaction after total knee arthroplasty: a realistic or imaginary goal? Orthop Clin North Am 48(4):421-431

17. Rodriguez-Merchan EC (2016) Patient dissatisfaction after total knee arthroplasty for hemophilic arthropathy and osteoarthritis (non-hemophilia patients). Expert Rev Hematol 9(1):59-68

18. Migliorini F et al (2019) Better outcomes with patellar resurfacing during primary total knee arthroplasty: a meta-analysis study. Arch Orthop Trauma Surg 139(10):1445-1454

19. Dean E (2016) Anxiety. Nurs Stand 30(46):15

20. Schuyler D (2016) Anxiety. Prim Care Companion CNS Disord. https://doi.org/10.4088/PCC.16f02039

21. Nechita D, Nechita F, Motorga R (2018) A review of the influence the anxiety exerts on human life. Rom J Morphol Embryol 59(4):1045-1051

22. APA. What is depression. 2017 https://www.psychiatry.org/patie nts-families/depression/what-is-depression.

23. Lim GY et al (2018) Prevalence of depression in the community from 30 countries between 1994 and 2014. Sci Rep 8(1):2861

24. Stubbs B et al (2016) Prevalence of depressive symptoms and anxiety in osteoarthritis: a systematic review and meta-analysis. Age Ageing 45(2):228-235

25. Mahdi A et al (2020) Patients' experiences of discontentment one year after total knee arthroplasty- a qualitative study. BMC Musculoskelet Disord 21(1):29

26. Tolk JJ et al (2019) Outcome expectations of total knee arthroplasty patients: the influence of demographic factors, pain, personality traits, physical and psychological status. J Knee Surg. https://doi.org/10.1055/s-0039-1692632

27. Lützner $C$ et al (2019) Fulfillment of expectations influence patient satisfaction 5 years after total knee arthroplasty. Knee Surg Sports Traumatol Arthrosc 27(7):2061-2070

28. Reichel F et al (2019) Predictors for persistent pain and dissatisfaction after total knee arthroplasty. Schmerz 33(3):185-190 
29. Bierke S, Haner M, Petersen W (2016) Influence of somatization and depressive symptoms on the course of pain within the first year after uncomplicated total knee replacement: a prospective study. Int Orthop 40(7):1353-1360

30. Bierke S, Petersen W (2017) Influence of anxiety and pain catastrophizing on the course of pain within the first year after uncomplicated total knee replacement: a prospective study. Arch Orthop Trauma Surg 137(12):1735-1742

31. Bistolfi A et al (2017) The presence and influence of mild depressive symptoms on post-operative pain perception following primary total knee arthroplasty. Knee Surg Sports Traumatol Arthrosc 25(9):2792-2800

32. Duivenvoorden T et al (2013) Anxiety and depressive symptoms before and after total hip and knee arthroplasty: a prospective multicentre study. Osteoarthritis Cartil 21(12):1834-1840

33. Gold HT et al (2016) Association of depression with 90-Day hospital readmission after total joint arthroplasty. J Arthroplasty 31(11):2385-2388

34. Wylde V et al (2017) Preoperative psychosocial risk factors for poor outcomes at 1 and 5 years after total knee replacement. Acta Orthop 88(5):530-536

35. Clement ND et al (2018) Patient age of less than 55 years is not an independent predictor of functional improvement or satisfaction after total knee arthroplasty. Arch Orthop Trauma Surg 138(12): 1755-1763

36. Collins NJ et al (2016) Knee injury and osteoarthritis outcome score (KOOS): systematic review and meta-analysis of measurement properties. Osteoarthr Cartil 24(8):1317-1329

37. Devlin NJ, Brooks R (2017) EQ-5D and the EuroQol Group: past, present and future. Appl Health Econ Health Policy 15(2):127-137

38. Djukanovic I, Carlsson J, Arestedt K (2017) Is the Hospital Anxiety and Depression Scale (HADS) a valid measure in a general population 65-80 years old? A psychometric evaluation study. Health Qual Life Outcomes 15(1):193

39. Stern AF (2014) The hospital anxiety and depression scale. Occup Med 64(5):393-394

40. Hyland KA et al (2019) Evaluation of the psychometric and structural properties of the Spanish Version of the hospital anxiety and depression scale in latina cancer patients. J Pain Symptom Manage 58(2):289-296

Publisher's Note Springer Nature remains neutral with regard to jurisdictional claims in published maps and institutional affiliations. 